CHD. 25 were simple or moderate complex non-cyanotic CHD. The estimated incidence was 1.828\%. $17 / 47$ (36.2\%) were cyanotic. The incidence of simple CHD was 25/47(53.2\%). Analysis of the specific anatomy is presented in table 1 .

\begin{tabular}{|c|c|c|}
\hline 1 & BAov (functional/anatomic) no additional disease & $3.035 \%$ \\
\hline 2 & VSD (isolated/all types) & $14.9 \%$ \\
\hline 3 & ASD (isolated/all types) & $8.5 \%$ \\
\hline 4 & PDA (isolated) & $6.384 \%$ \\
\hline 5 & C-AVSD & $4.258 \%$ \\
\hline 6 & A-P window & $2.128 \%$ \\
\hline 7 & Coronary artery abnormalities & $4.256 \%$ \\
\hline 8 & PA valve stenosis & $4.256 \%$ \\
\hline 9 & Ao valve stenosis & $4.256 \%$ \\
\hline 10 & Mitral valve disease & $2.128 \%$ \\
\hline 11 & CoA (isolated/all types) & $4.256 \%$ \\
\hline 12 & IAA (isolated/all types) & $2.128 \%$ \\
\hline 13 & ToF (all types) & $6.384 \%$ \\
\hline 14 & d-TGA (all types) & $4.256 \%$ \\
\hline 15 & PAv atresia with IVS & $4.256 \%$ \\
\hline 16 & DORV (all types) & $4.256 \%$ \\
\hline 17 & TAPVD (all types) & $4.256 \%$ \\
\hline 18 & Tricuspid valve atresia & $2.128 \%$ \\
\hline 19 & HLHS & $2.128 \%$ \\
\hline 20 & c-c-TGA & $2.128 \%$ \\
\hline 21 & Trancus Arteriosus & $2.128 \%$ \\
\hline 22 & Ebstein Anomaly & $2.128 \%$ \\
\hline 23 & Univentricular Anatomy & $2.128 \%$ \\
\hline 24 & Heterotaxia Syndromes & $2.128 \%$ \\
\hline 25 & Shone's Complex & $2.128 \%$ \\
\hline 26 & Core triatriatum & $2.128 \%$ \\
\hline
\end{tabular}

Conclusions This first-ever prospective epidemiology study in Azerbaijan involving a cohort equal to $1.65 \%$ of the annual living births of the country, estimated a high incidence. This is among the highest reported globally. The amount of critical $\&$ cyanotic CHD was $46.8 \%$ and $36.2 \%$ respectable. These high numbers are possibly related to an isolated population and conjugated marriage customs of the country. This represents a public population health burden.

\section{OC40 IS DOWN SYNDROME RELATED ARTHRITIS (DA) A DISTINCT DISEASE FROM JUVENILE IDIOPATHIC ARTHRITIS (JIA)?}

${ }^{1,2}$ Charlene Foley ${ }^{*},{ }^{1,2}$ Orla Killeen, ${ }^{1}$ Emma MacDermott, ${ }^{3}$ Gerry Wilson, ${ }^{4}$ Achilleas Floudas, ${ }^{4}$ Ursula Fearon. ${ }^{1}$ National Centre for Paediatric Rheumatology, Dublin, Ireland; ${ }^{2}$ National Children's Research Centre, Dublin, Ireland; ' ${ }^{3}$ niversity College Dublin, Dublin, Ireland; ${ }^{4}$ Trinity Biomedical Sciences Institute, Dublin, Ireland

10.1136/archdischild-2019-epa.39

DA is frequently polyarticular-RF-negative with predominance in the small joints of the hands and wrists. It has an erosive phenotype, and clinically appears to be a more aggressive disease than JIA. Arthritis occurs more frequently in children with Down syndrome (DS), with prevalence 20 times greater than JIA. To date little is known about the underlying mechanisms that drive DA disease pathogenesis, however these features suggest that DA may be distinct from JIA. We wanted to explore this hypothesis by examining B-cell subsets and T- cell cytokine profiles; and characterising and comparing the synovial membrane immunohistochemistry in DA versus JIA.

Methods Multicolour-flow cytometry was used to analyse B and $T$ cell phenotypes in PBMCs from 40 children $(n=10 /$ group - Healthy Control (HC), JIA, DS, DA), and assessed by Flowjo software analysis. Cells were stained as follows; B-cell panel (CD38, CD24, CD20, CD80, CD27, IgM, CD138, CD45, CD19, MHC class II, BCMA, CD40, CD86, IgD); Tcell panel - cytokines analysed after 5-hours PMA/Ionomycin stimulation (CD3, CD8, CD161, IFN- $\gamma$, TNF- $\alpha$, IL-17a, GMCSF).

Synovial tissue was obtained through US-guided biopsy and analysed by immunohistochemistry for CD3, CD20, CD68, FVIII (DA $n=3$; JIA $n=4$ ). Levels of vascularity and lining layer hyperplasia were also scored. Analysis was performed using a semi-quantification scoring method.

Results Flow cytometry analysis revealed that children with DA had a significantly lower number of circulating CD19 $+\mathrm{CD} 20+\mathrm{B}$ cells when compared to children with JIA $(p<0.05)$ and HC $(p<0.001)$. However, they had a greater proportion of memory $\mathrm{B}$ cells $(\mathrm{CD} 27+)$ when compared to children with DS $(\mathrm{p}<0.05)$.

IFN- $\gamma$ and TNF- $\alpha$ production by CD $8+/ C D 8-T$ cells was greater in DA compared to both JIA $(\mathrm{CD} 8+\mathrm{IFN} \gamma+\mathrm{p}<0.001$; $\mathrm{CD} 8+\mathrm{TNF} \alpha+\mathrm{p}<0.01 ; \mathrm{CD} 8-\mathrm{IFN} \gamma+\mathrm{p}<0.05 ; \mathrm{CD} 8-\mathrm{TNF} \alpha$ $\mathrm{p}<0.05)$ and HC $(\mathrm{CD} 8+\mathrm{IFN} \gamma+\mathrm{p}<0.05 ; \mathrm{CD} 8+\mathrm{TNF} \alpha+$ $\mathrm{p}<0.05$; CD8-IFN $\gamma+\mathrm{p}<0.05 ;$ CD8-TNF $\alpha \mathrm{p}<0.01)$.

Examination of synovial tissue demonstrated marked increase in synovial lining layer hyperplasia in DA (median 6 (3-9)) versus JIA (median 3(2-4)). Higher levels of CD3+ cells $(p<0.05)$, Macrophages $(p<0.05), \quad \mathrm{CD} 20+$ cells and FVIII in DA were also observed.

Conclusion Significant differences were observed in the immune and histological profiles of DA and JIA. These differences may begin to explain the erosive phenotype observed in DA. This study is important as it is the first of its kind to describe the immuno-histology of DA. Understanding the pathogenesis of DA will allow for more targeted, personalised treatment approaches, in turn improving outcomes and therefore quality of life for children with Down syndrome and arthritis.

\section{OC42 PHARMACOLOGICAL INDUCTION OF A20: ANTI- INFLAMMATORY EFFECTS IN PAEDIATRIC ASTHMA}

${ }^{1,2}$ Katy McConnell*, ${ }^{1,3}$ Gayathria Thillaiyampalam, ${ }^{1}$ Amal ElBanna, ${ }^{2}$ Arlene Brown, ${ }^{1}$ Madeleine Ennis, ${ }^{4,1}$ Shu-Dong Zhang, ${ }^{1,2}$ Michael Shields, 'Bettina Schock. 'Queen's University Belfast, Belfast, UK; ${ }^{2}$ Hospital for Sick Children, Paediatric Respiratory Clinic, Belfast, UK; ${ }^{3}$ University of Queensland, Brisbane, Australia; ${ }^{4}$ University of Ulster, Londonderry, UK

\subsection{6/archdischild-2019-epa.40}

Background Chronic exaggerated inflammation in asthma is mediated by the activation of pro-inflammatory transcription factor $\mathrm{NF}-\mathrm{kB}(\mathrm{p} 65)$ and an intrinsic lack of its down-regulator A20. A20 has strong anti-inflammatory effects when induced, and as such could be an attractive anti-inflammatory pharmacological target. Bioinformatic approaches using statistically significant connection Map (sscMap)) predict that prednisone and amitriptyline would induce A20 in asthmatic airway epithelial cells. We performed a proof of concept study to determine whether amitriptyline applied to nasal airway epithelial cells (AEC) from children could be beneficial in asthma. 
Methods We obtained nasal AECs by brush sampling from children with severe atopic asthma (AA) and healthy controls (HC) $(n=3-6)$. Cells were cultured in the presence/absence of the predicted drugs amitriptyline and prednisone and stimulated with lipopolysaccharide (LPS, $10 \mu \mathrm{g} / \mathrm{mL}, 0-24 \mathrm{~h}$ ), to mimic a bacterial infection. A20 and p65 mRNA and the release of pro-inflammatory cytokines from AEC cultures were determined.

Results AEC basal A20 was lower in AA compared to HC $(\mathrm{p}<0.05)$. LPS stimulation induced A20 in HC rapidly (peak at $1 \mathrm{~h}$ LPS, $\mathrm{p}<0.05$ ) and the elevated levels were maintained for up to 4 hours. In AA, LPS also caused an increase in A20 mRNA (lower than in $\mathrm{HC}$ ) and found to be only elevated at 4h. NF-kB p65 significantly increased $1 \mathrm{~h}$ after LPS stimulation in $\mathrm{HC}$ and $4 \mathrm{~h}$ after LPS in AA cells (both $\mathrm{p}<0.05$ ).

Amitriptyline (effective concentration $10 \mu \mathrm{M}$ ), increased A20 levels in both HC and AA epithelial cells. HC responded with a peak expression at $1 \mathrm{~h}$ LPS $(\mathrm{p}<0.05)$, while in AA cells, we observed a steady increase in A20 for up to 24h LPS. Prednisone $\left(10^{-3} \mu \mathrm{M}\right)$ induced A20 with a peak expression at $4 \mathrm{~h}$ in $\mathrm{AA}$ and $\mathrm{HC}$, but the increase was significantly higher in HC epithelial cells.

The increase in A20 mRNA was paralleled by a significant decrease in p65 mRNA in amitriptyline and prednisone-treated cells and a decrease in IL- 8 release.

Conclusion SscMap predicted drugs that successfully induced the anti-inflammatory protein A20 in AECs, which resulted in a reduced inflammatory response to bacterial stimulation (LPS). Although the anti-inflammatory effect of prednisone is well established, we here add that this is mediated through the induction of A20. Furthermore, the application of amitriptyline as an anti-inflammatory medication may need further investigation. This proof of concept study using bioinformatics could be used to identify other drugs that could be repositioned as anti-inflammatory treatment in asthma.

\section{OC43 IMPACT OF FLASH AND CONTINUOUS GLUCOSE MONITORING ON QUALITY OF LIFE AND DISEASE BURDEN IN CHILDREN WITH DIABETES MELLITUS AND THEIR FAMILIES}

Elaine Murphy*, Eleanor Burke, Laura McCarron, Gillian O'Donnell, Eirin Carolan, Ciara McDonnell, Nuala Murphy. Children's University Hospital Temple Street, Dublin, Ireland

\subsection{6/archdischild-2019-epa.41}

Introduction/Background Flash and continuous glucose monitoring technology has revolutionised the management of diabetes, particularly in paediatric populations. Recent government funding for flash glucose monitoring devices has enabled widespread usage in children and adolescents in the Republic of Ireland. We examined the impact of this novel technology on the lives of children with diabetes and their parents through the use of age modified questionnaires.

Design/Methods Qualitative research was carried out using anonymised 'Quality of Life' questionnaires. All children and adolescents and their families attending the diabetes service in Children's University Hospital were eligible for the study and were invited to contribute at routine outpatient visit. Those using the technology were divided into three groups; preschool, primary school and secondary school. Together, children and their parents answered questions relating to ease of use, impact on schooling, independence, parent-child relationships and subjective burden of disease. Some sections were specific to the particular form of glucose monitoring [flash or continuous]; but those regarding impact on day-today management of diabetes and impact on quality of life were common to all. A separate questionnaire was used for patients not using either technology and explored the factors that influenced that decision.

Results Preliminary data $(n=39)$ shows that patient and parent experiences with flash and continuous glucose monitoring technology have been overwhelmingly positive. 90\% of children surveyed are using one of these devices. Across all age groups, $100 \%$ of those using the technology reported subjective improvement in glycaemic control. All adolescents reported increased confidence in their ability to manage diabetes and a greater degree of personal independence. Parents reported significant reduction in frequency of hypoglycaemic events and $>80 \%$ reported reduction in anxiety surrounding hypoglycaemia. $>85 \%$ of both patients and parents found a significant reduction in the day-to-day burden of diabetes. Those not using glucose-monitoring devices listed parental concern regarding device accuracy and attachment of the device on the skin as the major deterring factors.

Conclusions This data demonstrates a positive impact of flash and continuous glucose monitoring on quality of life for children with diabetes mellitus. Government funding since 2018 has enabled equal access for all paediatric patients [over 4 years old] to this life-impacting technology which should now be offered to all children and adolescents, to include those with Type 2 Diabetes and other rarer sub-types, and those under 4 years old.

\section{OC44 OPTIMIZATION OF DIAGNOSTICS FOR CARBOHYDRATE MECHANISM DISORDER IN CHILDREN WITH CYSTIC FIBROSIS}

${ }^{1}$ Tatiyana Matveyeva, ${ }^{2}$ Aleksandr Orlov, ${ }^{1}$ Marina Tkach, ${ }^{1}$ Liudmila Zhelenina, ${ }^{3}$ Mariya Kuropatenko, 'Liliya Ditkovskaya*. ${ }^{1}$ St.Petersburg State Pediatric Medical University, Saint Petersburg, Russian Federation; ${ }^{2}$ St.Olga Children's Hospital, Saint Petersburg, Russian Federation; ${ }^{3}$ FSBSI 'Institute of Experimental Medicine', Saint Petersburg, Russian Federation

\subsection{6/archdischild-2019-epa.42}

Aim To study the incidence and optimize diagnostics of carbohydrate mechanism disorder in children with cystic fibrosis (CF).

Materials and methods We have examined 43 patients with cystic fibrosis. All the patients were subject to the following tests: blood chemistry, HbA1c, insulin, C-peptide EIA assay, antibodies to ICA, IAA, GAD in order to rule out other forms of diabetes mellitus, oral glucose tolerance test (GTT). Carbohydrate mechanism disorders were diagnosed according to the WHO criteria (ISPAD 2018). In order to assess tissue sensitivity to insulin, Homa and Caro indices were calculated during the study. Additionally, 31 patients (including 15 children aged 3-11, others were adolescents) underwent Continuous Glucose Monitoring System (CGMS) test with the help of MiniMed Paradigm 722. All the children were given a molecular genetic test in order to detect various GFTR mutations.

Results The oral glucose tolerance test revealed carbohydrate metabolism disorders in $28 \%$ of the case, CF-associated diabetes mellitus was diagnosed in $19 \%$ of the case. Impaired glucose tolerance was diagnosed in $9 \%$ patients. Carbohydrate metabolism disorders among adolescents were distributed as 